\title{
Noninvasive continuous monitoring of cardiovascular dynamics during complex endoscopic procedures to improve patient safety
}

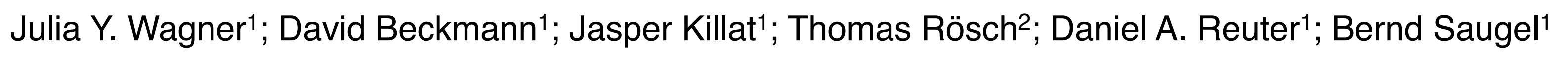

\author{
${ }^{1}$ Department of Anesthesiology, Center of Anesthesiology and Intensive Care Medicine, ${ }^{2}$ Department of \\ Interdisciplinary Endoscopy, University Medical Center Hamburg-Eppendorf, Martinistrasse 52, 20246 Hamburg, \\ Germany
}

\begin{abstract}
Introduction: During endoscopic procedures, at least intermittent arterial pressure (AP) measurements and pulse oximetry are required for patient monitoring. However, studies have shown that patients with obstructive jaundice have an increased sensitivity to sedative agents with more hypotension and bradycardia and prolonged recovery time after anesthesia. The $\mathrm{CNAP}^{\circledR}$ technology (CNSystems Medizintechnik AG, Graz, Austria) enables continuous noninvasive AP (CNAP) waveform recordings based on the volume clamp method. In this study we investigated whether the use of noninvasive continuous AP monitoring might help to detect hypotensive episodes earlier and more often in patients undergoing endoscopic retrograde cholangiopancreatography (ERCP) or percutaneous transhepatic cholangiography and drainage (PTCD) in comparison with intermittent oscillometrically derived AP measurements.
\end{abstract}

\begin{tabular}{|l|l|}
\hline number of patients, $\mathrm{n}$ & 77 \\
\hline male, $\mathrm{n}(\%)$ & $45(58)$ \\
\hline female, $\mathrm{n}(\%)$ & $32(42)$ \\
\hline age, years & $61( \pm 16)$ \\
\hline BMI, $\mathrm{kg} / \mathrm{m}^{2}$ & $24( \pm 5)$ \\
\hline ERCP, $\mathrm{n}(\%)$ & $66(86)$ \\
\hline PTCD, $\mathrm{n}(\%)$ & $11(14)$ \\
\hline
\end{tabular}

Results: Sixty-six (86\%) and $11(14 \%)$ of the study patients underwent ERCP and PTCD, respectively. The mean duration of the procedure was $37( \pm 22)$ min. All patients were sedated with propofol. The mean AP for CNAP and oscillometry were $93 \pm 26$ and $97 \pm 16 \mathrm{~mm} \mathrm{Hg}$ for the mean AP and $122 \pm 34$ and $127 \pm 21 \mathrm{~mm} \mathrm{Hg}$ for the systolic AP, respectively. In the interval between two oscillometric measurements, one or more hypotensive phases were detected in 25 patients for mean AP and 28 patients for systolic AP.

\begin{abstract}
Methods: The study patients' AP was simultaneously monitored intermittently at 5-minute intervals using upper arm cuff oscillometry and continuously using CNAP during the complete endoscopic procedure. Seventy-seven patients were analyzed in this interim analysis. Hypotension was defined as an AP $>10 \%$ below the last measured oscillometric value and $\leq 65 \mathrm{~mm} \mathrm{Hg}$ for the mean $\mathrm{AP}$ and $\leq$ $90 \mathrm{~mm} \mathrm{Hg}$ for the systolic AP. A hypotensive phase measured by CNAP in the interval between two intermittent AP measurements was defined as a time period of at least 30 seconds during which $>50 \%$ of the AP values were in the defined range of hypotension.
\end{abstract}

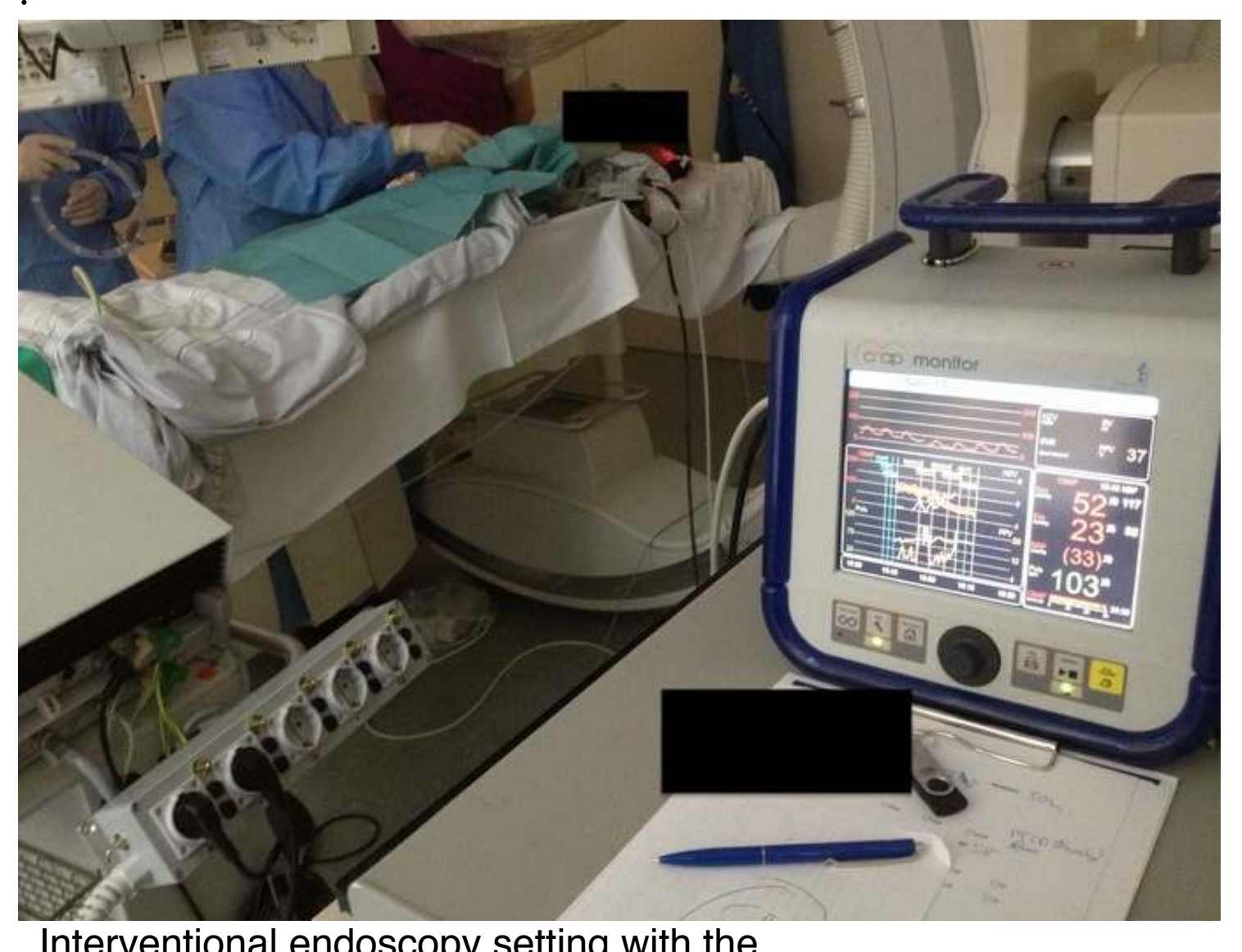

Interventional endoscopy setting with the CNAP monitor in the right corner.

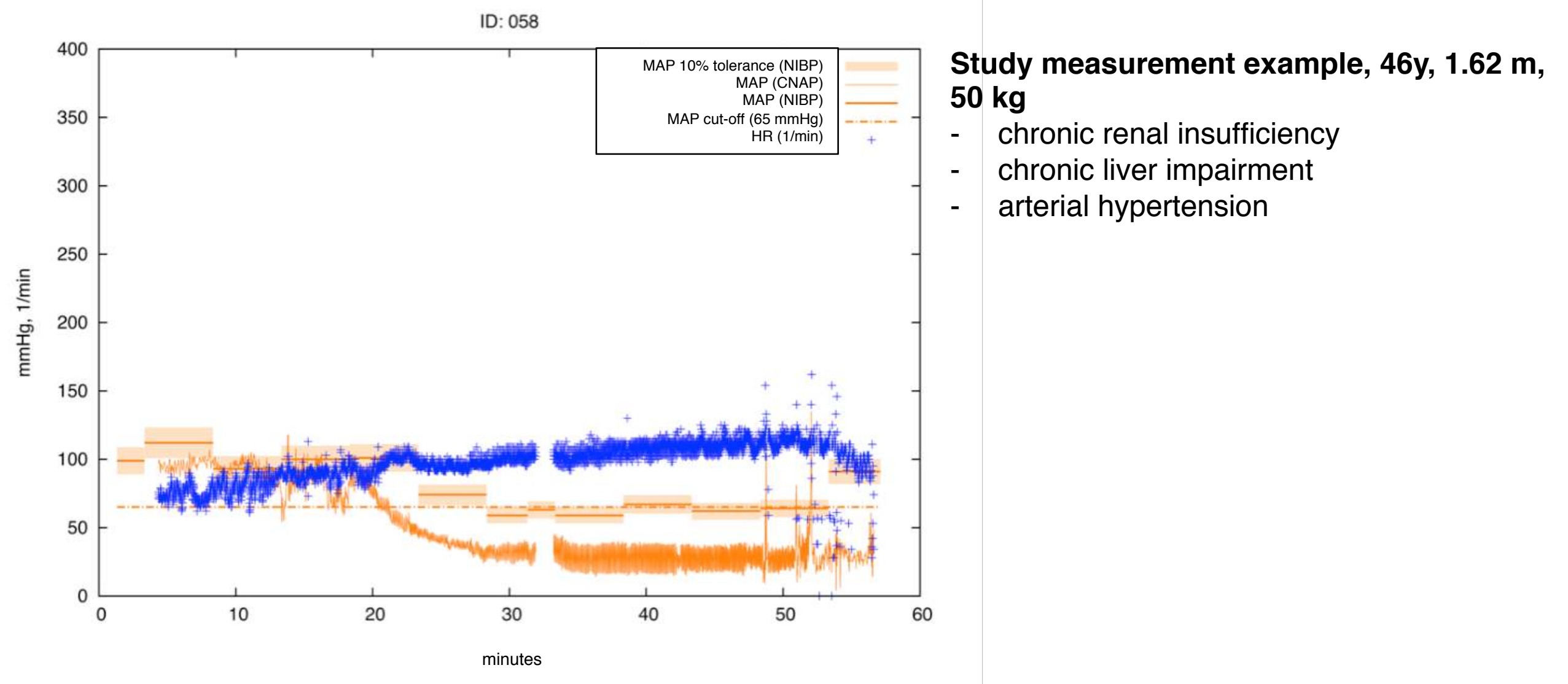

Conclusion: By the earlier detection of hypotensive phases in patients undergoing complex endoscopic procedures, continuous noninvasive monitoring of cardiovascular dynamics allows faster treatment of hemodynamic instability and might therefore increase patient safety.

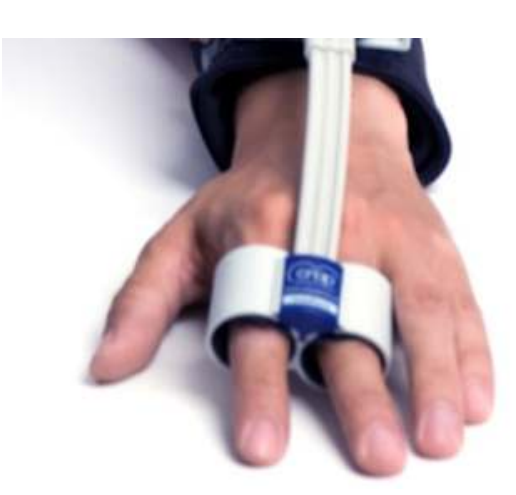

CNAP double finger cuff (www.cnsystems.de) 